\title{
Room Access Control System Using Facial Image Recognition Resmana Lim ${ }^{1,2}$, a Frans Rotinsuluand ${ }^{1, b}$ and Petrus Santoso ${ }^{1, c}$ ${ }^{1}$ Electrical Engineering Dept., Petra Christian University - Indonesia \\ ${ }^{2}$ Center of Excellence Geopolymer \& Green Technology (CEGeoGTech), School of Materials Engineering, Universiti Malaysia Perlis, Malaysia \\ aresmana@petra.ac.id, ${ }^{b}$ m23411016@john.petra.ac.id, ${ }^{c}$ petrus@petra.ac.id
}

Keywords: access control, face recognition, home automation, computer vision

\begin{abstract}
The aim of the project is to implement a facial recognition system for access control to enter a room. The facial image captured by a webcam and then be detected/tracked using Haar face tracking algorithm. Principal Component Analysis (PCA) and Linear Discriminant Analysis (LDA) algorithm have been used for face recognition. The system was tested with 10 users from the member of a laboratory room. Each user registered 100 images for training of the PCA and LDA. The recognition rate achieved using PCA was 70\% and 97\% for LDA.
\end{abstract}

\section{Introduction}

Face recognition system has advantages compared with other biometric systems such as using fingerprints/palm and iris, because the process is non-contact. Facial image can be captured from a distance without touching the person being identified, and the identification does not need to interact with the person. In addition, the facial recognition deterrent purpose of the crime because pictures of faces that have been recorded and archived and can help identify a person [1].

PCA and LDA method has been widely used in face recognition and has been implemented in the OpenCV library [2]. In particular the LDA method has been used for student attendance system [3]. The combined method of PCA and LDA can achieve an accuracy of 99\% [4].

Our project has been implemented for access control in a laboratory room. Only registered members may access to the room. Training faces with different expressions and poses processed by PCA and LDA to be used to recognized a face input from a webcam.

\section{Methods and System Set-up}

Figure 1 shows the system set-up of our room access conrtol based on facial recognition. When the user approaches to the camera, a Pasive Infra Red (PIR) sensor will detect the presence and began to turn on the LED lights. These lights are used to assure that sufficient illumination so that the camera can capture images properly. The software will process the image with face tracking and face image croping to do recognition using PCA and LDA. Only registered members have access entrance into the room. We utilize Open source Computer Vision (OpenCV) library and EmguCV especially in Haar face detection/tracking, and image recognition by PCA and LDA. 


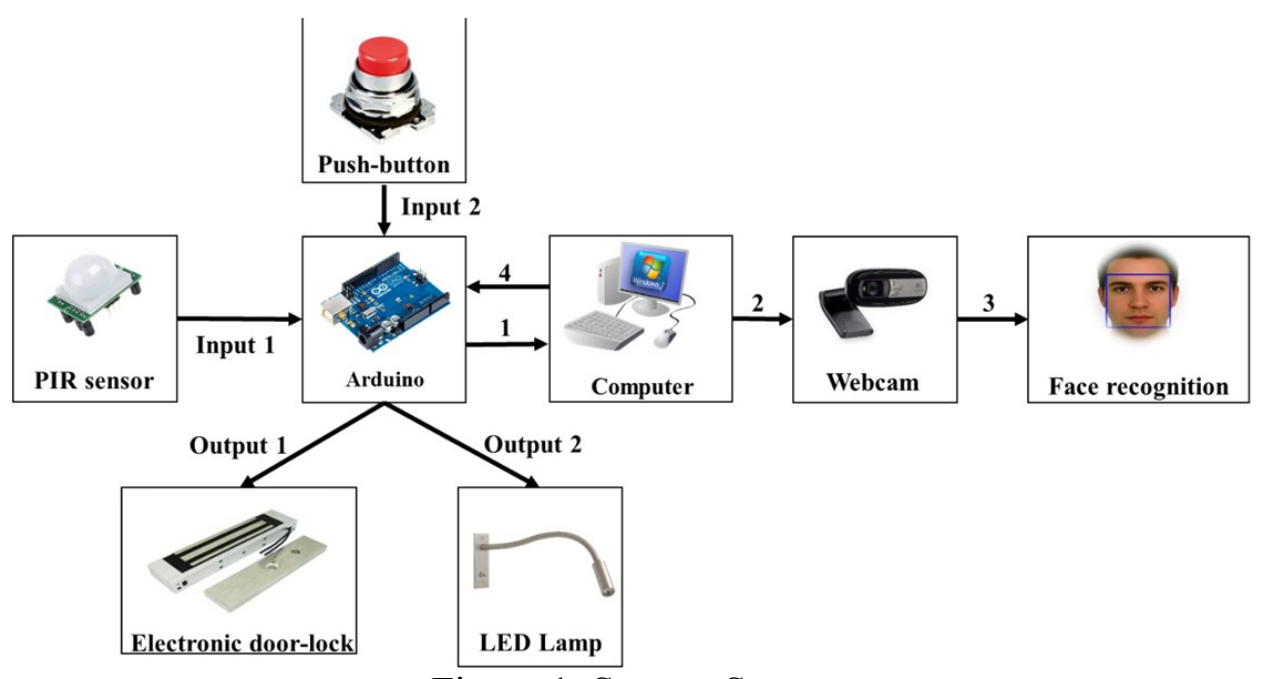

Figure 1. System Set-up

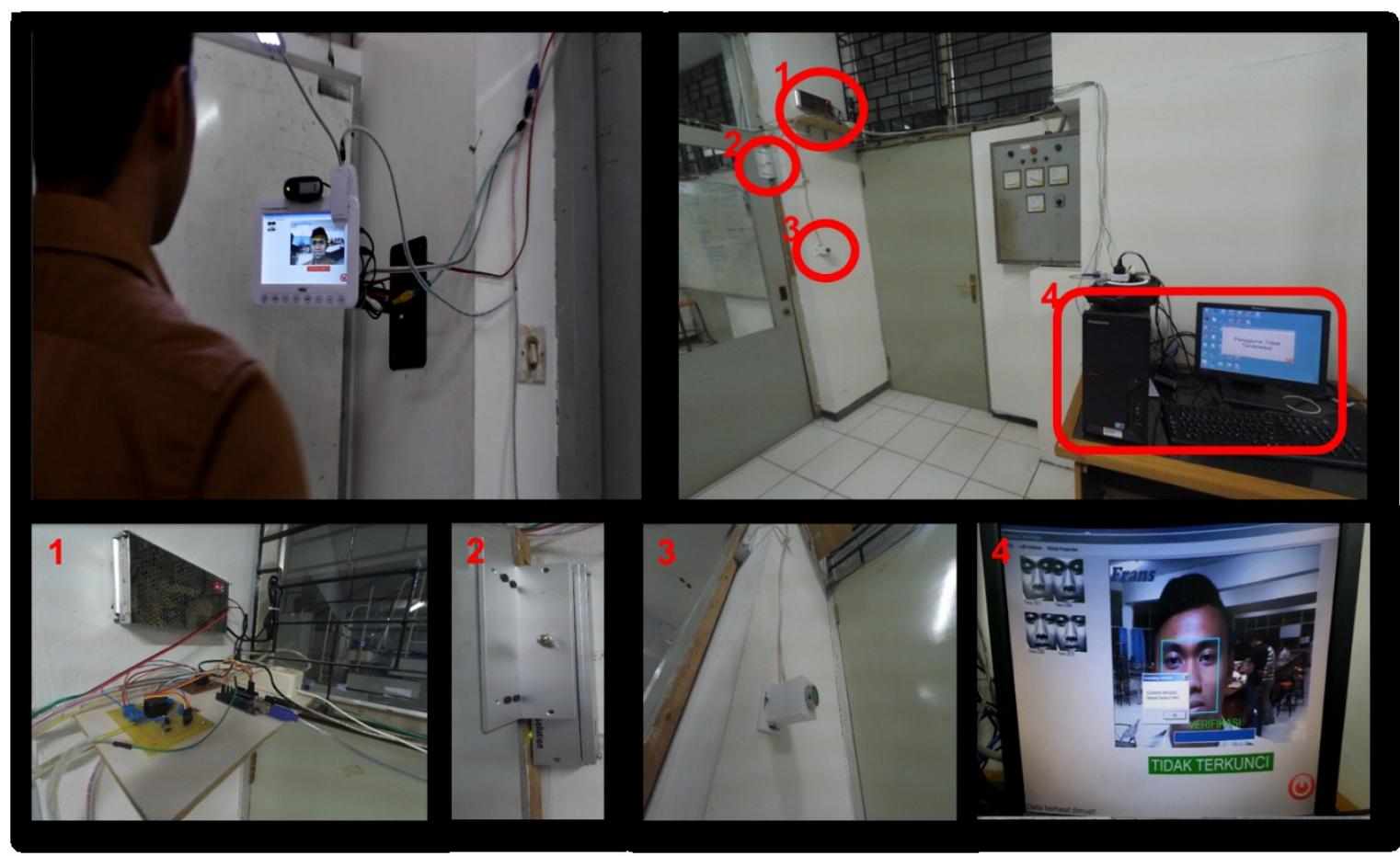

Figure 2. System implementation

\section{Haar Face Tracking}

We use Haar wavelets which called as Haar-like features [5]. It already implemented on OpenCV library. The Haar detection tracks facial image, and capture face images for recognition purposes. Haar cascade needs to be trained. It needs positive and negative samples. Positive samples are images contain of faces (object of interest) and negative samples are images contain of background, such as wall, floor, etc.

\section{Principal Component Analysis (PCA) [6.7]}

A 2D image with dimensions $\mathrm{b}$ rows and $\mathrm{k}$ columns can be represented in the form of a 1D image with dimensions of $n(n=b * k)$. Training images is used as indicated by $\left\{x_{1}, x_{2}, \ldots, x_{K}\right\}$ consist of the $\mathrm{K}$ samples taken from $c$ objects/classes represented by $\left\{X_{1}, X_{2}, \ldots, X_{c}\right\}$. Total scatter matrix $S_{T}$ (or covariance matrix) is defined as follows:

$$
S_{T}=\sum_{k=1}^{K}\left(x_{k}-\mu\right)\left(x_{k}-\mu\right)^{T}
$$


Where $\mu$ is the average sample image obtained by averaging the image training $\left\{x_{1}, x_{2}, \ldots, x_{K}\right\}$. With eigen decomposition, this covariance matrix can be decomposed into:

$$
S_{T}=\Phi \Lambda \Phi^{T}
$$

Where $\Phi$ is the eigenvector matrix, and $\Lambda$ is is a diagonal matrix of eigenvalues. Then select a number of $m$ columns of the matrix eigenvector associated with the largest number of $m$ eigenvalues. This eigenvector election produces a transformation matrix or projection matrix of $\Phi_{m}$, which is composed of $m$ column that was chosen eigenvectort that commonly referred to as the 'eigenimage'. Next an image $x$ (with dimension of $n$ ) can be extracted into a new feature of $y$ (with dimension $m<n$ ) by projecting the $x$ with the direction of $\Phi_{m}$ as the following:

$$
y=\Phi_{m} x
$$

In other words PCA is projecting the space of origin $\mathfrak{R}^{n}$ into a new lower space dimension of $\mathfrak{R}^{m}$, where as much as possible the original information content will be retained for not too much disappeared after being taken to a smaller feature dimensions [8,9]. Here we can see a significant reduction feature of $n$ into $m$ pieces which will greatly ease the computing of the recognition process in the next phase.

Total scatter matrix $S_{T}$ above is actually the sum of a within-class scatter matrix $S_{W}$ and the inter-class scatter matrix (between-class scatter matrix) $S_{B}$, ie, $S_{T}=S_{W}+S_{B}$. Thus, the major drawback seen here is that in the process of PCA, the two scatter matrix are maximized together. Indeed desired is only maximization of $S_{B}$ alone, whereas minimized $S_{W}$ as much as possible so that members distribution can be collected in the class, which in turn can increase the success rate of the recognition. Suppose that the variation of illumination change and the scale of the image that occurs on the same object, can cause matrix becomes large scatter in the classroom is quite difficult in the recognition process. When this occurs, thereby PCA will include a variety of illumination in its eigenimage, and consequently PCA be reliable enough against illumination variations that occur in the object. With the LDA method, $S_{W}$ will be minimized so that the resulting feature extraction becomes more reliable against variations that occur in the class [10].

\section{Linear Discriminant Analysis (LDA)}

Within-class scatter matrix $S_{W}$, and the inter-class (between-class) scatter matrix $S_{B}$ is defined respectively as follows $[4,10]$ :

$$
\begin{gathered}
S_{W}=\sum_{i=1}^{c} \sum_{x_{k} \in X_{i}}\left(x_{k}-\mu_{i}\right)\left(x_{k}-\mu_{i}\right)^{T} \\
S_{B}=\sum_{i=1}^{c} N_{i}\left(\mu_{i}-\mu\right)\left(\mu_{i}-\mu\right)^{T}
\end{gathered}
$$

Which $N_{i}$ is the number of samples in the class of $X_{i}$, and $\mu_{i}$ is the average image of the class $X_{i}$. As stated previously that it is expected that the within-class scatter matrix $S_{W}$ can be minimized while inter-class scatter matrix $S_{B}$ is maximized. In other words, the projection matrix $\Psi_{l}$ will be sought so that the ratio equation (6) becomes maximal.

$$
\frac{\operatorname{det}\left(\Psi_{l} S_{B} \Psi_{l}^{T}\right)}{\operatorname{det}\left(\Psi_{l} S_{W} \Psi_{l}^{T}\right)}
$$

These criteria resulted in a solution with the following equation [3]:

$$
S_{B} \Psi=S_{W} \Psi \Lambda
$$


Where $\Psi$ is the eigenvector matrix, and $\Lambda$ is the diagonal matrix of eigenvalues. In other words to be searched eigenvector and eigenvalue of the matrix $C$ which is a combination within and between class scatter matrix as in eq. 8. Again, the election of as many as $l$ columns of the eigenvector $\Psi$ associated with the largest eigenvalues. Selection of column eigenvector $l$ produces a projection matrix $\Psi_{l}$ that is subsequently used for feature extraction as well as on the PCA [4,10].

$$
C=S_{b} S_{w}^{-1}
$$

\section{Results and Discussion}

The system was tested with the users of $10(c=10)$ laboratory staffs who are allowed to access the room. Each staff has 100 images with different poses and expressions, so the total we have K of 1000 sample/training images $(K=10 \times 100=1000)$. Each image was in grayscale with the size of $100 \times 100$ pixels. These images have been used as training images for PCA (with the size of feature $m=80$ components) and LDA.

Table 1. Recognition rate using PCA method

\begin{tabular}{|c|c|c|c|c|}
\hline Number & $\begin{array}{c}\text { Staff } \\
\text { Name }\end{array}$ & Total Detected & Correct & Recognition Rate (\%) \\
\hline 1 & Andry & 16 & 16 & 100 \\
\hline 2 & Kevin & 53 & 0 & 0 \\
\hline 3 & Edus & 171 & 50 & 29,24 \\
\hline 4 & Frans & 16 & 16 & 100 \\
\hline 5 & Kimsha & 18 & 17 & 94,44 \\
\hline 6 & Lau & 18 & 17 & 94,44 \\
\hline 7 & Vincent & 53 & 40 & 75,47 \\
\hline 8 & Wili & 16 & 16 & 100 \\
\hline 9 & Yefta & 45 & 0 & 0 \\
\hline 10 & Youngky & 16 & 16 & $\mathbf{6 9 , 3 6 \%}$ \\
\hline
\end{tabular}

Table 2. Recognition rate using LDA method

\begin{tabular}{|c|c|c|c|c|}
\hline Number & $\begin{array}{c}\text { Staff } \\
\text { Name }\end{array}$ & Total Detected & Correct & Recognition Rate (\%) \\
\hline 1 & Brian & 17 & 17 & 100 \\
\hline 2 & Daniel & 19 & 19 & 100 \\
\hline 3 & Frans & 23 & 22 & 95,65 \\
\hline 4 & Hans & 16 & 16 & 100 \\
\hline 5 & Kevin & 17 & 17 & 100 \\
\hline 6 & Lau & 16 & 16 & 100 \\
\hline 7 & Mettu & 16 & 16 & 100 \\
\hline 8 & Vincent & 17 & 16 & 94,12 \\
\hline 9 & William & 16 & 16 & 85,71 \\
\hline 10 & Youngky & 21 & 18 & $\mathbf{9 7 , 5 5}$ \\
\hline
\end{tabular}

We tested the method of PCA and LDA for all staffs by approaching webcam to enter the room. Several images of each person have been detected/taken with different expressions and poses and then be tested to recognized. Tables 1 and 2 show the performance results of PCA and LDA respectively. LDA algorithm is superior and more suitable to be used on the access control. 


\section{Conclusion}

The access control system based on face recognition has been implemented. The accuracy of the recognition using PCA was $70 \%$ and $97 \%$ for LDA. From the experimental results, in general, when a variety of training images is high enough (eg: illumination, expression and pose) then the use of LDA will contribute higher recognition rate. Even with a smaller number of features, LDA gives better results when in comparison with the use of PCA.

\section{References}

[1] A. K. Jain, A. Ross, and S. Prabhakar: An introduction to biometric recognition, Circuits and Systems for Video Technology, IEEE Transactions on 14(1), (2004) p. 4-20.

[2] A. C. Bhensle and R. Raja, An efficient face recognition using PCA and Euclidean distance classification. International Journal of Computer Science and Mobile Computing, 3(6), (2014) p. 407-413.

[3] W. Budhiharto, The access control system based on linear discriminant analysis. Journal of Computer Science, 10(3), (2014) p. 453-457. doi:10.3844/jcssp.2014.453.457

[4] S. Singh, M. Sharma and S. Rao, Accurate face recognition using PCA and LDA. International Journal of Computer Science and Information Security, 10(3), (2012) p. 103-112.

[5] J. Cho, S. Mirzaei, J. Oberg and R. Kastner, in: FPGA-Based face detection system using Haar classifiers ,Proceedings of the ACM/SIGDA international symposium on Field programmable gate arrays. ACM, (2009)

[6] M. P. Shermi and A. M. Ali (2012). A principal component analysis method for recognition of human faces: Eigenfaces approach. International Journal of Electronics Communication and Computer Technology (IJECCT), 2(3), p. 139-144.

[7] L. C. Paul, and A. A. Sumam, Face recognition using principal component analysis method. International Journal of Advanced Research in Computer Engineering \& Technology (IJARCET), 1(9), (2012) p. 135-139.

[8] C. Johnson, EMGU Multiple Face Recognition using PCA and Parallel Optimisation $\begin{array}{lllll}\text { CodeProject. } & \text { Retrieved } & \text { May } & \text { 13, 2015, from }\end{array}$ http:/www.codeproject.com/Articles/261550/EMGU-Multiple-Face-Recognition-using-PCA-a nd-Paral

[9] Liliana, R. Lim, and E. Kwan, in: Voice conversion application (VOCAL). Uncertainty Reasoning and Knowledge Engineering (URKE), International Conference on. Vol. 1. IEEE, (2011).

[10] K. Etemad and R. Chellappa: Discriminant Analysis for Recognition of Human Face Images, Journal of Optical Society of America A, (1997) pp. 1724-1733. 